\title{
A General Synthesis of Substituted Indoles from Cyclic Enol Ethers and Enol Lactones
}

Kevin R. Campos*, Jacqueline C. S. Woo, Sandra Lee and Richard D. Tillyer

Department of Process Research, Merck \& Co., Inc., PO Box 2000, Rahway, NJ 07065-0900.

\section{Supporting Information}

Analytical methods: All reactions were carried out under nitrogen. Flash chromatography was carried out with EM science Silica gel 60 (neutral, 230-400 mesh). ${ }^{1} \mathrm{H}$ NMR and ${ }^{13} \mathrm{C}$ NMR spectra were recorded on a Bruker Avance 400 NMR Spectrometer with chemical shifts reported in ppm relative to the residual deuterated solvent. IR spectra were recorded on a Nicolet Margna FT-IR Spectrometer 560. HPLC analysis was performed on a Hewlett Packard 1100 MSD instrument using a Waters Symmetry C18 4.6mm x $250 \mathrm{~mm}$ column and acetonitrile/0.4\% $\mathrm{HClO}_{4}$ as eluents. HRMS was recorded on a Micromass QT of Ultima API US mass spectrometer by ESI.

\section{General procedure for the Fischer indole reaction:}
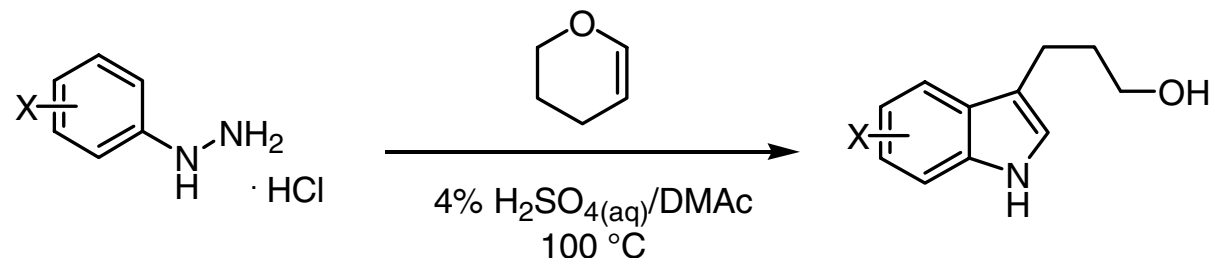

To a solution of phenyhydrazine- $\mathrm{HCl}(1 \mathrm{~g}, 6.92 \mathrm{mmol})$ in $4 \% \mathrm{H}_{2} \mathrm{SO}_{4(\mathrm{aq})}(10 \mathrm{~mL})$ and $N, N$-dimethyl acetamide (DMAc, $10 \mathrm{~mL}$ ) at $100{ }^{\circ} \mathrm{C}$ was added dihydrofuran (630 uL, $6.92 \mathrm{mmol}$ ) dropwise over 2 minutes. The reaction was aged for 2 hours, then cooled to room temperature, extracted with ethyl acetate $(20 \mathrm{~mL})$ and washed with water $(20 \mathrm{~mL}) 3$ times. The crude material was purified by flash chromatography.

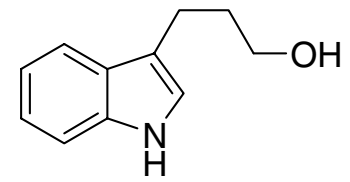

3-(1H-indol-3-yl)propan-1-ol (2a): Synthesized according to the general procedure to afford 2a in $90 \%$ isolated yield after chromatography. Pale yellow oil, IR (neat) 3544, 3408, 2953, 2876, 1456, 1338, 1054; ${ }^{1} \mathrm{H}$ NMR (400 MHz, $\left.\mathrm{CDCl}_{3}\right) \delta 7.96($ br s, 1H), $7.63(\mathrm{dt}, 1 \mathrm{H}, \mathrm{J}=8.0,1.2 \mathrm{~Hz}$ ), 7.37 (dt, 1H, J=8.0, $0.8 \mathrm{~Hz}), 7.21$ (ddd, 1H, $\mathrm{J}=8.0,7.2,1.2 \mathrm{~Hz}), 7.13(\mathrm{dd}, 1 \mathrm{H}, \mathrm{J}=8.0,1.2 \mathrm{~Hz}), 7.01(\mathrm{~d}, 1 \mathrm{H}, \mathrm{J}=0.8 \mathrm{~Hz}), 3.75(\mathrm{t}, 2 \mathrm{H}, \mathrm{J}=6.4 \mathrm{~Hz}), 2.88$ 
(dt, J=7.6, $0.8 \mathrm{~Hz}, 2 \mathrm{H}), 2.01(\mathrm{~m}, 2 \mathrm{H}) ;{ }^{13} \mathrm{C}$ NMR 136.3, 127.4, 121.9, 121.2, 119.1, 118.8, 115.9, 111.0, $62.6,32.8,21.3$.

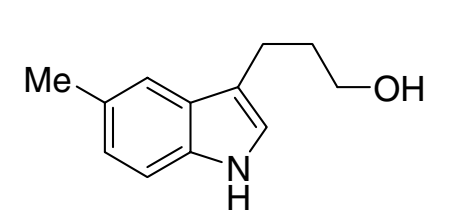

3-(5-methyl-1H-indol-3-yl)propan-1-ol (2b): Synthesized according to the general procedure to afford $\mathbf{2 b}$ in $95 \%$ isolated yield after chromatography. Pale yellow oil, IR (neat) 3407, 2936, 2858, 1481, 1440, 1228, 1055, 1054; ${ }^{1} \mathrm{H}$ NMR (400 MHz, CDCl $)$ $\delta 7.96$ (br s, 1H), 7.45 (s, 1H), 7.25 (d, 1H, J=8.4 Hz), 7.07 (dd, 1H, J=8.4, $1.2 \mathrm{~Hz}), 6.92(\mathrm{~d}, 1 \mathrm{H}, \mathrm{J}=0.8 \mathrm{~Hz}), 3.75$ (t, 2H, J=6.4 Hz), 2.86 (dt, J=7.6, $0.8 \mathrm{~Hz}, 2 \mathrm{H}), 2.52$ (s, 3H), 2.01 (m, 2H); ${ }^{13}$ C NMR 136.1, 127.1, 122.6, 121.0, 120.3, 119.5, 116.7, 116.6, 62.8, 33.1, 21.6, 16.6.

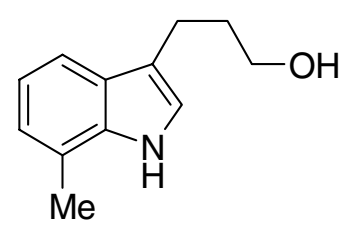

3-(7-methyl-1H-indol-3-yl)propan-1-ol (2e): Synthesized according to the general procedure to afford $\mathbf{2 e}$ in $71 \%$ isolated yield after chromatography. White solid, m.p. $=95.0{ }^{\circ} \mathrm{C}$, IR (neat) $3526,3243,2920,2850,1448,1233,1050 ;{ }^{1} \mathrm{H}$ NMR (400 $\left.\mathrm{MHz}, \mathrm{CDCl}_{3}\right) \delta 7.91($ br s, 1H), $7.49(\mathrm{~d}, 1 \mathrm{H}, \mathrm{J}=8.0 \mathrm{~Hz}), 7.04(\mathrm{~m}, 3 \mathrm{H}), 3.75(\mathrm{t}, 2 \mathrm{H}$, $\mathrm{J}=6.4 \mathrm{~Hz}), 2.88(\mathrm{t}, \mathrm{J}=7.6 \mathrm{~Hz}, 2 \mathrm{H}), 2.50(\mathrm{~s}, 3 \mathrm{H}), 2.01(\mathrm{~m}, 2 \mathrm{H}) ;{ }^{13} \mathrm{C}$ NMR 136.1, 127.1, 122.6, 121.0, $120.3,119.5,116.7,116.6,62.8,33.1,21.6,16.6$.

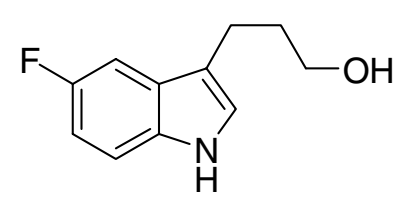

3-(5-fluoro-1H-indol-3-yl)propan-1-ol (2f): Synthesized according to the general procedure to afford $\mathbf{2 f}$ in $90 \%$ isolated yield after chromatography. Pale yellow oil, IR (neat) 3423, 2922, 2880, 1581, 1485, 1230, 1171, 1055; ${ }^{1} \mathrm{H}$ $\operatorname{NMR}\left(400 \mathrm{MHz} \mathrm{CDCl}_{3}\right) \delta 8.22($ br s, 1H), $7.26(\mathrm{dd}, 1 \mathrm{H}, \mathrm{J}=7.6,2.4 \mathrm{~Hz}), 7.23(\mathrm{~m}, 1 \mathrm{H}), 6.99(\mathrm{~s}, 1 \mathrm{H})$, $6.95(\mathrm{dt}, 1 \mathrm{H}, \mathrm{J}=9.2,2.4 \mathrm{~Hz}), 3.72(\mathrm{t}, 2 \mathrm{H}, \mathrm{J}=6.4 \mathrm{~Hz}), 2.80(\mathrm{dt}, \mathrm{J}=7.6,0.8 \mathrm{~Hz}, 2 \mathrm{H}), 1.97(\mathrm{~m}, 2 \mathrm{H}) ;{ }^{13} \mathrm{C}$ NMR 158.7, 156.4, 132.8, 127.8, 127.7, 123.2, 115.9, 115.8, 111.7, 110.2, 110.0, 103.7, 103.5, 62.4, $32.7,21.2$.

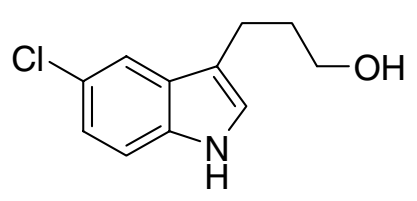

3-(5-chloro-1H-indol-3-yl)propan-1-ol (2g): Synthesized according to the general procedure except that acetonitrile was used as cosolvent instead of DMAc. Purification by chromatography afford $\mathbf{2 g}$ in $70 \%$ isolated yield. Pale yellow oil, IR (neat) 3572, 3427, 2925, 2854, 1462, 1227, 1099, 1056; ${ }^{1} \mathrm{H} \mathrm{NMR}\left(400 \mathrm{MHz}, \mathrm{CDCl}_{3}\right) \delta$ 8.01 (br s, 1H), 7.58 (d, 1H, J=2.0 Hz), 7.26 (d, 1H, J=2.4 Hz), 7.15 (dd, 1H, J=8.4, 2.4 Hz), 7.03 (m, 1H), $3.74(\mathrm{t}, 2 \mathrm{H}, \mathrm{J}=6.4 \mathrm{~Hz}), 2.83(\mathrm{dt}, \mathrm{J}=7.6,0.8 \mathrm{~Hz}, 2 \mathrm{H}), 1.98(\mathrm{~m}, 2 \mathrm{H}) ;{ }^{13} \mathrm{C} \mathrm{NMR} 134.6,128.5,124.9$, $122.6,122.2,118.3,115.7,112.0,62.4,32.8,21.1$. 


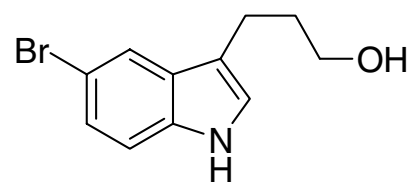

3-(5-bromo-1H-indol-3-yl)propan-1-ol (2h): Synthesized according to the general procedure except that acetonitrile was used as cosolvent instead of

DMAc. Purification by chromatography afforded $\mathbf{2 h}$ in $72 \%$ isolated yield. Pale yellow oil, IR (neat) 3425, 2919, 2879, 1458, 1094, 1055; ${ }^{1} \mathrm{H}$ NMR $\left(400 \mathrm{MHz}, \mathrm{CDCl}_{3}\right) \delta 7.95$ (br s, 1H), $7.61(\mathrm{~d}, 1 \mathrm{H}, \mathrm{J}=1.8 \mathrm{~Hz}), 7.14(\mathrm{dd}, 1 \mathrm{H}, \mathrm{J}=8.6,1.8 \mathrm{~Hz}), 7.09$ (dd, 1H, J=8.6, 0.8, $1.2 \mathrm{~Hz}), 6.87$ (s, 1H), 3.59 (t, 2H, J=6.4 Hz), 2.67 (dt, J=7.6, $0.8 \mathrm{~Hz}, 2 \mathrm{H}), 1.83(\mathrm{~m}, 2 \mathrm{H}) ;{ }^{13} \mathrm{C}$ NMR 134.9, 129.2, 124.7, $122.5,121.4,115.6,112.5,112.4,62.4,32.8,21.1$.

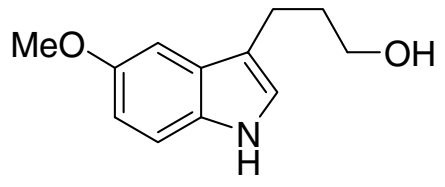

3-(5-methoxy-1H-indol-3-yl)propan-1-ol (2i): Synthesized according to the general procedure except that the reaction was performed at $60{ }^{\circ} \mathrm{C}$ for 3 hours. Purification by chromatography afforded $\mathbf{2 i}$ in $75 \%$ isolated yield. Pale yellow oil, IR (neat) 3417, 2936, 1623, 1583, 1455, 1291, 1032; ${ }^{1} \mathrm{H}$ NMR (400 MHz, $\left.\mathrm{CDCl}_{3}\right) \delta$ 7.91 (br s, 1H), 7.25 (d, 1H, J=8.8 Hz), 7.06 (d, 1H, J=2.0 Hz), 6.98 (s, 1H), 6.87 (dd, 1H, J=8.4, 7.4 $\mathrm{Hz}), 3.88$ (s, 3H), 3.75 (t, 2H, J=6.4 Hz), $2.84(\mathrm{dt}, \mathrm{J}=7.6,0.8 \mathrm{~Hz}, 2 \mathrm{H}), 2.00(\mathrm{~m}, 2 \mathrm{H}) ;{ }^{13} \mathrm{C}$ NMR 153.9, $131.8,128.0,122.4,115.7,112.2,112.0,101.0,62.7,56.2,32.9,21.5$.

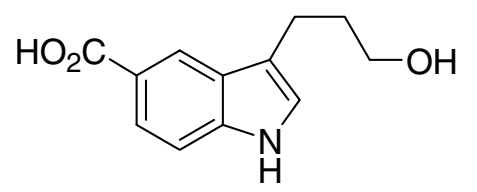

3-(3-hydroxypropyl)-1H-indole-5-carboxylic acid (2j): white solid, m.p. $=165.7^{\circ} \mathrm{C}$, IR (neat) $3373,2926,1673,1615,1579,1445,1324,1248$; ${ }^{1} \mathrm{H}$ NMR (400 MHz, CD OD) $\delta 8.33(\mathrm{dd}, 1 \mathrm{H}, \mathrm{J}=1.6,0.8 \mathrm{~Hz}), 7.77$ (dd, 1H, $\mathrm{J}=8.4,1.6 \mathrm{~Hz}), 7.34(\mathrm{dd}, 1 \mathrm{H}, \mathrm{J}=8.4,0.8 \mathrm{~Hz}), 7.10(\mathrm{t}, 1 \mathrm{H}, \mathrm{J}=0.8 \mathrm{~Hz}), 3.61(\mathrm{t}, 2 \mathrm{H}, \mathrm{J}=6.4 \mathrm{~Hz}), 2.83(\mathrm{dt}, \mathrm{J}$ = 7.6, $0.8 \mathrm{~Hz}, 2 \mathrm{H}), 1.93(\mathrm{~m}, 2 \mathrm{H}) ;{ }^{13} \mathrm{C} \mathrm{NMR}$ 170.2, 139.4, 126.9, 123.0, 122.3, 121.4, 120.2, 116.3, $110.3,61.1,32.8,20.7$.

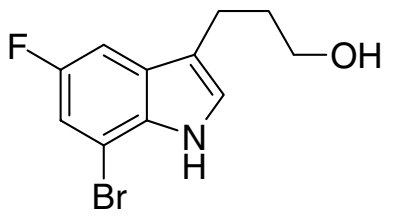

3-(7-bromo-5-fluoro-1H-indol-3-yl)propan-1-ol (2k): Synthesized according to the general procedure to afford $\mathbf{2 k}$ in $60 \%$ isolated yield after chromatography. White solid, m.p. $=61.9{ }^{\circ} \mathrm{C}$, IR (neat) 3424, 2929, 2855, 1571, 1489, 1305, 1160, 1053; ${ }^{1} \mathrm{H}$ NMR (400 MHz, $\mathrm{CDCl}_{3}$ ) $\delta 8.31$ (br s, 1H), 7.22 (dd, 1H, J=9.2, 2.0 Hz), $7.15(\mathrm{dd}, 1 \mathrm{H}, \mathrm{J}=8.4,2.0 \mathrm{~Hz}), 7.06$ (s, 1H), 3.72 (t, 2H, J=6.4 Hz), 2.78 (dt, J=7.6, 0.8 Hz, 2H), 1.95 (m, 2H); ${ }^{13} \mathrm{C}$ NMR 158.3, 155.9, 132.0, 128.3, 128.2, 123.9, 117.6, 117.5, 113.2, 112.9, 104.2, $104.1,103.5,103.3,62.4,32.8,21.4$. 
$\mathrm{SO}_{2} \mathrm{NHMe}$

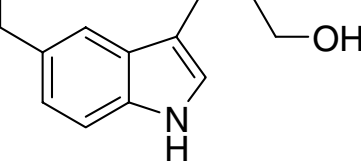

literature.

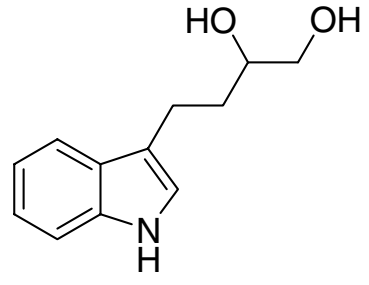

1-[3-(3-hydroxypropyl)-1H-indol-5-yl]-N-methylmethanesulfonamide

(4):

Synthesized according to general procedure except that acetonitrile was used as cosolvent instead of DMAc. Purification by chromatography afforded 4 in $50 \%$ isolated yield. All spectral data matched that which is reported in the (dd, 1H, J=11.2, 3.2 Hz), 3.50 (dd, 1H, J=11.2, 7.2 Hz), 2.94 (sept, 1H, J=7.2 Hz), 2.90 (sept, 1H, $\mathrm{J}=7.2 \mathrm{~Hz}), 1.88(\mathrm{~m}, 2 \mathrm{H}) ;{ }^{13} \mathrm{C}$ NMR $136.5,127.5,122.1,121.4,119.3,118.9,115.9,111.2,71.9$, 66.9, $33.4,21.2$

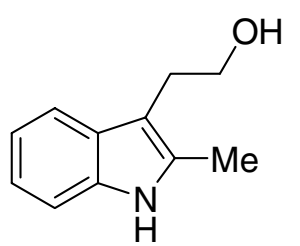

2-(2-methyl-1H-indol-3-yl)ethanol (10): Synthesized according to the general procedure to afford 9 in $80 \%$ isolated yield after chromatography. Pale yellow oil, IR (neat) 3399, 2933, 1462, 1300, 1043; ${ }^{1} \mathrm{H} \mathrm{NMR}\left(400 \mathrm{MHz}, \mathrm{CDCl}_{3}\right) \delta 7.96$ (br s, 1H), $7.55(\mathrm{~d}, 1 \mathrm{H}, \mathrm{J}=7.2 \mathrm{~Hz}), 7.27(\mathrm{~d}, 1 \mathrm{H}, \mathrm{J}=7.6 \mathrm{~Hz}), 7.15(\mathrm{~m}, 2 \mathrm{H}), 3.85(\mathrm{t}, 2 \mathrm{H}$, $\mathrm{J}=6.4 \mathrm{~Hz}), 2.99(\mathrm{t}, \mathrm{J}=6.8 \mathrm{~Hz}, 2 \mathrm{H}), 2.38(\mathrm{~s}, 2 \mathrm{H}) ;{ }^{13} \mathrm{C}$ NMR 135.5, 132.6, 128.8, 121.2, 119.4, 118.0, $110.4,107.6,62.9,27.8,11.7$.

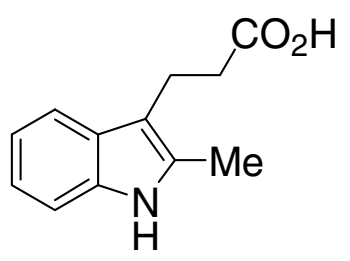

3-(2-methyl-1H-indol-3-yl)propanoic acid (12): Synthesized according to the general procedure to afford $\mathbf{1 1}$ in $75 \%$ isolated yield after chromatography. White solid, m.p.=133.8 ${ }^{\circ} \mathrm{C}$; IR (neat) 3373, 2926, 1673, 1615, 1445, 1324, 1248; ${ }^{1} \mathrm{H}$ NMR (400 MHz, CD OD) $\delta 7.43(\mathrm{~d}, 1 \mathrm{H}, \mathrm{J}=7.6 \mathrm{~Hz}), 7.22(\mathrm{dd}, 1 \mathrm{H}$, $\mathrm{J}=7.6,1.2 \mathrm{~Hz}), 7.02(\mathrm{dt}, 1 \mathrm{H}, \mathrm{J}=7.6,1.2 \mathrm{~Hz}), 6.99(\mathrm{~m}, 2 \mathrm{H}), 3.00(\mathrm{t}, 2 \mathrm{H}, \mathrm{J}=7.6 \mathrm{~Hz}), 2.56(\mathrm{t}, 2 \mathrm{H}, \mathrm{J}=7.6$ $\mathrm{Hz}), 2.32$ (s, 3H); ${ }^{13} \mathrm{C}$ NMR 176.2, 135.6, 131.3, 128.1, 119.9, 118.1, 117.0, 109.9, 108.9, 34.8, 19.5, 9.9 .

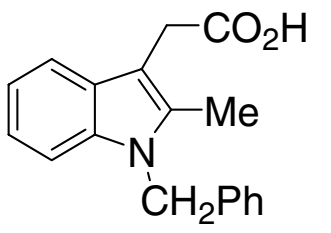

(1-benzyl-2-methyl-1H-indol-3-yl)acetic acid (16): Synthesized according to general procedure except that acetonitrile was used as cosolvent instead of DMAc, and the reaction was aged for 24 hour to affect complete conversion. Assay of the entire reaction mixture to a pure sample determined the yield to be $70 \%$. After the 
standard workup, 15 could be isolated via crystallization from 70:30 hexane:ethyl acetate as a white solid, m.p. $=175{ }^{\circ} \mathrm{C}$, IR (neat) 3028, 2920, 1699, 1469, 1358, 1299; ${ }^{1} \mathrm{H}$ NMR (400 MHz, CD $\mathrm{ODD} \delta$ $7.54(\mathrm{~m}, 1 \mathrm{H}), 7.25(\mathrm{~m}, 4 \mathrm{H}), 7.07(\mathrm{dq}, 1 \mathrm{H}, \mathrm{J}=7.2,1.6 \mathrm{~Hz}), 6.99(\mathrm{~m}, 2 \mathrm{H}), 5.41(\mathrm{~s}, 2 \mathrm{H}), 3.76(\mathrm{~s}, 2 \mathrm{H}), 2.36$ (s, 3H); ${ }^{13} \mathrm{C}$ NMR 177.0, 140.4, 138.6, 130.3, 130.0, 128.8, 127.8, 122.6, 120.8, 119.6, 110.8, 106.7, $31.7,10.9$.

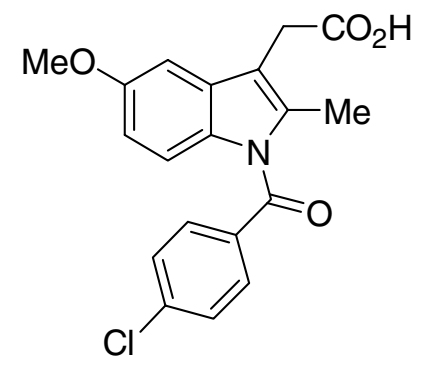

[1-(4-chlorobenzoyl)-5-methoxy-2-methyl-1H-indol-3-yl]acetic acid (18): Synthesized according to general procedure except that acetonitrile was used as cosolvent instead of DMAc, and the reaction was aged for 24 hour to affect complete conversion. Assay of the entire reaction mixture compared to an authentic sample determined the yield of $\mathbf{1 8}$ to be $65 \%$.

\section{References:}

1. Brodfuehrer, P. R.; Chen, B.-C.; Sattelberg, T. R.; Smith, P. R.; Reddy, J. P.; Stark, D. R.; Quinlan, S. L.; Reid, J. G.; Thottathil, J. K.; Wang, S. J. Org. Chem. 1997, 62, 9192-92022. 